\title{
Sympathetic Nervous System
}

National Cancer Institute

\section{Source}

National Cancer Institute. Sympathetic Nervous System. NCI Thesaurus. Code C12795.

The part of the autonomic nervous system comprising paired, paraspinal sympathetic trunks together with their branches, plexi, and subsidiary gang lia; it functions to mobilize the body for activity and opposes the physiological effects of the parasympathetic nervous system. 